\title{
Recognition of Alzheimer's Dementia From the Transcriptions of Spontaneous Speech Using fastText and CNN Models
}

\author{
Amit Meghanani, C. S. Anoop* and Angarai Ganesan Ramakrishnan \\ MILE Laboratory, Department of Electrical Engineering, Indian Institute of Science, Bengaluru, India
}

Alzheimer's dementia (AD) is a type of neurodegenerative disease that is associated with a decline in memory. However, speech and language impairments are also common in Alzheimer's dementia patients. This work is an extension of our previous work, where we had used spontaneous speech for Alzheimer's dementia recognition employing log-Mel spectrogram and Mel-frequency cepstral coefficients (MFCC) as inputs to deep neural networks (DNN). In this work, we explore the transcriptions of spontaneous speech for

OPEN ACCESS

Edited by:

Saturnino Luz,

University of Edinburgh,

United Kingdom

Reviewed by:

Diego R. Amancio,

University of São Paulo, Brazil

Anna Pribilova,

Slovak Academy of Sciences (SAS),

Slovakia

${ }^{*}$ Correspondence:

C. S. Anoop

anoopcs@iisc.ac.in

Specialty section:

This article was submitted to

Human-Media Interaction,

a section of the journal

Frontiers in Computer Science

Received: 31 October 2020

Accepted: 25 January 2021

Published: 24 March 2021

Citation:

Meghanani A, Anoop CS and Ramakrishnan AG (2021) Recognition

of Alzheimer's Dementia From the

Transcriptions of Spontaneous

Speech Using fastText and

CNN Models.

Front. Comput. Sci. 3:624558

doi: 10.3389/fcomp.2021.624558 dementia recognition and compare the results with several baseline results. We explore two models for dementia recognition: 1) fastText and 2) convolutional neural network $(\mathrm{CNN})$ with a single convolutional layer, to capture the n-gram-based linguistic information from the input sentence. The fastText model uses a bag of bigrams and trigrams along with the input text to capture the local word orderings. In the CNN-based model, we try to capture different n-grams (we use $n=2,3,4,5$ ) present in the text by adapting the kernel sizes to $\mathrm{n}$. In both fastText and CNN architectures, the word embeddings are initialized using pretrained GloVe vectors. We use bagging of 21 models in each of these architectures to arrive at the final model using which the performance on the test data is assessed. The best accuracies achieved with CNN and fastText models on the text data are 79.16 and $83.33 \%$, respectively. The best root mean square errors (RMSE) on the prediction of mini-mental state examination (MMSE) score are 4.38 and 4.28 for CNN and fastText, respectively. The results suggest that the n-gram-based features are worth pursuing, for the task of $A D$ detection. fastText models have competitive results when compared to several baseline methods. Also, fastText models are shallow in nature and have the advantage of being faster in training and evaluation, by several orders of magnitude, compared to deep models.

Keywords: fastText, convolutional neural network, Alzheimer's, dementia, mini-mental state examination

\section{INTRODUCTION}

Dementia is a syndrome characterized by the decline in cognition that is significant enough to interfere with one's independent, daily functioning. Alzheimer's disease contributes to around $60-70 \%$ of dementia cases. Toward the final stages of Alzheimer's dementia (AD), the patients lose control of their physical functions and depend on others for care. As there are no curative treatments for dementia, the early detection is critical to delay or slow down the onset or progression of the 
disease. The mini-mental state examination (MMSE) is a widely used test to screen for dementia and to estimate the severity and progression of cognitive impairment.

$\mathrm{AD}$ affects the temporal characteristics of spontaneous speech. Changes in the spoken language are evident even in mild $\mathrm{AD}$ patients. Subtle language impairments such as difficulties in word finding and comprehension, usage of incorrect words, ambiguous referents, loss of verbal fluency, speaking too much at inappropriate times, talking too loudly, repeating ideas, and digressing from the topic are common in the early stages of AD (Savundranayagam et al., 2005) and they turn extreme in the moderate and severe stages. Szatlóczki et al. (2015) show that AD can be detected with the help of a linguistic analysis more sensitively than with other cognitive examinations. Mueller et al. (2018b) analyzed the connected language samples obtained from simple picture description tasks and found that the speech fluency and the semantic content features declined faster in participants with early mild cognitive impairment. The language profile of $\mathrm{AD}$ patients is characterized by "empty speech," devoid of content words (Nicholas et al., 1985). They tend to use pronouns without proper noun references and indefinite terms like "this," "that," and "thing" more often (Mueller et al., 2018a). These results motivate us to believe that modeling the transcriptions of the narrative speech in the cookie-theft picture description task using n-gram language models can help in the detection of $\mathrm{AD}$ and prediction of MMSE score.

In this work we address the $\mathrm{AD}$ detection and MMSE score prediction problems using two natural language processing (NLP)-based models: 1) fastText and 2) convolutional neural network $(\mathrm{CNN})$. These models have the advantage that they can be easily structured to capture the linguistic cues in the form of n-grams from the transcriptions of the picture description task, provided with the Alzheimer's Dementia Recognition through Spontaneous Speech (ADReSS) dataset (Luz et al., 2020). CNNs, though originated in computer vision, have become popular for NLP tasks and have achieved great results in sentence classification (Kim, 2014), semantic parsing (tau Yih et al., 2014), search query retrieval (Shen et al., 2014), and other traditional NLP tasks (Collober et al., 2011). Our convolutional neural network model draws inspiration from the work on sentence classification using CNNs (Kim, 2014). The fastText (Joulin et al., 2017) is a simple and efficient model for text classification (e.g., tag prediction and sentiment analysis). The fundamental idea in the fastText classifier is to calculate the n-grams of an input sentence and append them to the end of the sentence. Our choice of fastText model is also motivated by its ability to often outperform deep learning classifiers in terms of accuracy and training/evaluation times (Joulin et al., 2017).

The rest of the paper is organized as follows. Section 2 discusses the ADReSS dataset in detail. Section 3 discusses the baseline results in $\mathrm{AD}$ detection. Section 4 discusses our proposed NLP-based models followed by the listing of results in Section 5. Our results and conclusions are discussed in Section 6.

\section{ADRESS DATASET}

The ADReSS dataset (Luz et al., 2020) is designed to provide Alzheimer's research community with a standard platform for
AD detection and MMSE score prediction. The dataset is acoustically preprocessed and balanced in terms of age and gender. It consists of audio recordings and transcriptions [in CHAT format (Macwhinney, 2009)] of the cookie-theft picture description task, elicited from subjects in the age group of 50-80 years. The training set consists of data from 108 subjects, 54 each from $\mathrm{AD}$ and non-AD classes. The test set has data from 48 subjects, again balanced with respect to $\mathrm{AD}$ and non- $\mathrm{AD}$ classes. More information on the ADReSS dataset can be found in the ADReSS challenge baseline paper (Luz et al., 2020).

\section{REVIEW OF BASELINE METHODS}

This section provides a brief overview of the various approaches for $\mathrm{AD}$ detection and MMSE score prediction on ADReSS dataset. These approaches can be broadly classified into three types based on the type of the features used in the problem: 1) acoustic feature, 2) linguistic feature, and 3) a fusion of acoustic and linguistic features. The performance of different approaches on the $\mathrm{AD}$ detection and MMSE score prediction tasks are compared using the accuracy and root mean square error (RMSE) measures computed on the ADReSS test set.

$$
\begin{gathered}
\text { Accuracy }=\frac{T N+T P}{N} \\
\mathrm{RMSE}=\sqrt{\frac{\sum_{i=1}^{N}\left(\hat{y}_{i}-y_{i}\right)^{2}}{N}}
\end{gathered}
$$

where $N$ is the total number of subjects involved in the study, TP the number of true positives, and $T N$ the number of true negatives. $\widehat{y}_{i}$ and $y_{i}$ are the estimated and target MMSE scores for $i^{\text {th }}$ test sample. The results of different approaches on the ADReSS dataset are summarized in Table 1.

\subsection{Acoustic Feature-Based Methods}

Luz et al. (2020) explore several acoustic features like extended Geneva minimalistic acoustic parameter set (eGeMAPS) (Eyben et al., 2016), emobase, ComParE-2013 (Eyben et al., 2013), and multiresolution cochleagram (MRCG) (Chen et al., 2014), feeding the traditional machine learning algorithms like linear discriminant analysis, decision trees, nearest neighbor, random forests, and support vector machines. In our previous work (Meghanani et al., 2021), we have used CNN/ResNet + long short-term memory (LSTM) networks and pyramidal bidirectional LSTM + CNN networks trained on log-Mel spectrogram and Mel-frequency cepstral coefficient (MFCC) features extracted from the spontaneous speech. Pompili et al. (2020) exploit the pretrained models to produce i-vector- and $\mathrm{x}$-vector-based acoustic feature embeddings. They evaluate $\mathrm{x}$-vector, i-vector, and statistical speech-based functional features. Rhythmic features are proposed in Campbell et al. (2020), as lower speaking fluency is a common pattern in patients with AD. Koo et al. (2020) use VGGish (Hershey et al., 2017) trained with Audio Set (Gemmeke et al., 2017) for audio classification. They have proposed a modified version of convolutional recurrent neural network (CRNN), where an 
TABLE 1 | Baseline methods on ADReSS test set.

\begin{tabular}{|c|c|c|}
\hline Model & Accuracy (\%) & RMSE \\
\hline Searle et al. (2020), DistilBERT & 81.25 & 4.58 \\
\hline Searle et al. (2020), SVM + CRF & 81.25 & 5.22 \\
\hline Pompili et al. (2020), x-vectors SRE & 54.17 & - \\
\hline Pompili et al. (2020), sentence embedding & 72.92 & - \\
\hline Pompili et al. (2020), fusion of system & 81.25 & - \\
\hline Luz et al. (2020), linguistic & 75.00 & 5.20 \\
\hline Sarawgi et al. (2020b), ensemble & 83.33 & 4.60 \\
\hline Koo et al. (2020), VGGish & 72.92 & 5.07 \\
\hline Koo et al. (2020), Transformer-XL & 81.25 & 4.01 \\
\hline Koo et al. (2020), VGGish + GloVe & 77.08 & 4.33 \\
\hline Koo et al. (2020), VGGish + transformer-XL & 75.00 & 3.74 \\
\hline Koo et al. (2020), ensembled output & 81.25 & 3.77 \\
\hline Campbell et al. (2020), fusion II & 75.00 & - \\
\hline Campbell et al. (2020), fusion I & 72.92 & - \\
\hline Campbell et al. (2020), RNN model & 75.00 & - \\
\hline Campbell et al. (2020), fluency & 60.42 & - \\
\hline Campbell et al. (2020), x-vector & 54.17 & - \\
\hline Sarawgi et al. (2020a), UA ensemble & - & 4.35 \\
\hline Sarawgi et al. (2020a), UA ensemble (weighted) & - & 3.93 \\
\hline Pappagari et al. (2020), acoustic and transcript & 75.00 & 5.37 \\
\hline Rohanian et al. (2020), LSTM (Lexical + Dis) & 72.92 & 4.88 \\
\hline Rohanian et al. (2020), LSTM with gating (Acoustic + Lexical) & 77.08 & 4.57 \\
\hline Rohanian et al. (2020), LSTM with gating (Acoustic + Lexical + Dis) & 79.17 & 4.54 \\
\hline Yuan et al. (2020), ERNIE3p & 89.58 & - \\
\hline Syed et al. (2020) & 85.42 & 4.30 \\
\hline Edwards et al. (2020), phonemes and audio & 79.17 & - \\
\hline Meghanani et al. (2021), CNN-LSTM with MFCC & $64 . .58$ & 6.24 \\
\hline Meghanani et al. (2021), pBLSTM-CNN with log-Mel & 52.08 & 5.90 \\
\hline Meghanani et al. (2021), ResNet-LSTM with log-Mel & 62.50 & 5.98 \\
\hline
\end{tabular}

attention layer is the forefront layer of the network, and fully connected layers follow the recurrent layer.

\subsection{Linguistic Feature-Based Methods}

Recently, there have been multiple attempts on the AD detection problem based on text-based features and models. Searle et al. (2020) use traditional machine learning techniques like support vector machines (SVMs), gradient boosting decision trees (GBDT), and conditional random fields (CRFs). They also try deep learning transformer-based models, specifically, bidirectional encoder representations from transformers (BERT) (Devlin et al., 2019), RoBERTa (Liu et al., 2019), and DistilBERT/DistilRoBERTa (Sanh et al., 2019). Pompili et al. (2020) encode each word of the clean transcriptions into 768dimensional context embedding vector using a frozen English BERT model pretrained with 12 layers. Three different neural models are trained on top of contextual word embeddings: 1) global maximum pooling, 2) bidirectional long short-term memory (BLSTM)-based recurrent neural networks (RNN) provided with an attention module, and 3) the second model augmented with part-of-speech (POS) embeddings. In the work of Campbell et al. (2020), authors have used the manual transcripts to extract linguistic information (interventions, vocabulary richness, frequency of verbs, nouns, POS-tagging, etc.) for creating the input features of the classifier. They use another sequential deep learning-based classifier, which directly classifies the sequence of Gobal Vectors (GloVe)-based word embeddings. Koo et al. (2020) use transformer-based language models (Vaswani et al., 2017), generative pretraining (GPT) (Radford et al., 2018), RoBERTa (Liu et al., 2019), and transformer-XL (Dai et al., 2020) to get textual features and perform classification and regression tasks using a modified convolutional recurrent neural network-based structure.

Graph-based representation of word features (Tomás and Radev, 2012; Cong and Liu, 2014), which have shown promise in classifying texts (De Arruda et al., 2016), is also employed for detection of mild cognitive impairments. Santos et al. (2017) model transcripts as complex networks and enrich them with word embedding to better represent short texts produced in neuropsychological assessments. They use metrics of topological properties of complex networks in a machine learning classification approach to distinguish between healthy subjects and patients with mild cognitive impairments. Such graph-based techniques have also been used in the word sense disambiguation (WSD) tasks to identify the meaning of words in a given context for specific words conveying multiple meanings. Corra et al. (2018) suggest that a bipartite network model with local features employed to characterize the context can be useful in improving the semantic characterization of written texts without the use of deep linguistic information.

\subsection{Bimodal Methods}

Methods with bimodal input features (both acoustic and linguistic) are also used for $\mathrm{AD}$ recognition in various studies 
(Sarawgi et al., 2020a; Sarawgi et al., 2020b; Campbell et al., 2020; Koo et al., 2020; Pompili et al., 2020; Rohanian et al., 2020). However, in this work, we restrict ourselves to the NLP-based approaches.

\section{PROPOSED NLP-BASED METHODS}

\subsection{Data Preparation}

In this work, we explore the linguistic features for $\mathrm{AD}$ detection and hence only the textual transcripts in the ADReSS dataset are used. The transcripts contain the conversational content between the participant and the investigator. This includes pauses in speech, laughter, and discourse markers such as "um" and "uh." Each transcript is considered as a single data point with their corresponding AD label and MMSE score. We create two transcription level datasets after preprocessing the transcripts as in Searle et al. (2020)-1) PAR: containing the utterances of participant alone, 2) PAR + INV: containing utterances from both the participant and the investigator. In addition to the preprocessing performed in Searle et al. (2020), we keep PAR and INV tags as well in the data (which defines whether the utterance is spoken by the participant or the investigator).

\subsection{Convolutional Neural Network Model}

Language impairments like difficulties in lexical retrieval, loss of verbal fluency, and breakdown in comprehension of higher order written and spoken languages are common in AD patients. Hence the linguistic information, like the n-grams present in the input sentence, may provide good cues for AD detection. Any $n \times d$ CNN filter, where $n$ is the number of sequential words looked over by the filter and $d$ is the dimension of word embedding, can be viewed as a feature detector looking for a specific n-gram in the input that can capture the language impairments associated with $\mathrm{AD}$.

We describe the details of the CNN model from the work (Kim, 2014) as follows. Let $z_{i} \in R^{d}$ be a $d$-dimensional word vector corresponding to the $i$ th word in the sentence. A sentence of length $L$ is represented as $\left\{z_{1}, z_{2}, \ldots, z_{L}\right\}$. Let $z_{i: i+j}$ represent the concatenation of the words $z_{i}, z_{i+1}, \ldots, z_{i+j}$. A convolution operation involves a filter $w \in R^{\text {nd }}$, which is applied to a window of $n$ words to produce a new feature as shown in Eq. 3, where $s_{i}$ is generated from a window of words $z_{i: i+n-1}$ by

$$
s_{i}=f\left(w \cdot z_{i: i+n-1}+b\right) .
$$

In Eq. 3, $f$ is a nonlinear function and $b$ is the bias term. A feature map $\mathcal{E}$ is obtained by applying the filter to all possible windows of words in the sentence $\left[z_{1: n}, z_{2: n+1}, \ldots, z_{L-n+1: L}\right]$.

$$
\mathcal{E}=\left[s_{1}, s_{2}, \ldots, s_{L-n+1}\right] .
$$

A max-pool over time (Collober et al., 2011) is performed over the feature map to get $s_{\max }=\max \mathcal{E}$ as the feature corresponding to that filter. This corresponds to the n-gram that is "most relevant" in the $\mathrm{AD}$ recognition task. The weights of the filters, which in turn determine the "most relevant" feature, are learnt using backpropagation. CNNs are trained with just one layer of convolution. Variable length sentences are automatically handled by the pooling scheme. We use pretrained 100dimensional GloVe word vectors (Pennington et al., 2014) for word embedding. Multiple kernels of sizes $2 \times 100,3 \times 100$, $4 \times 100$, and $5 \times 100$ are employed to have a look at the bigrams, trigrams, 4-grams, and 5-grams within the text. We use 100 filters each with heights 2, 3, 4, and 5. Multiple configurations with filter sizes $[2,3,4],[3,4,5]$, and $[2,3,4,5]$ are applied which are referred to as $\mathrm{CNN}-b i+t r i+4$ gram, $\mathrm{CNN}$ tri $+4+5$ gram, and CNN-bi+tri+4+5 gram in our tables. The outputs of the filter are concatenated together to form a single vector. Dropout with probability $p=0.5$ is applied on the concatenated filter output and the results are passed through a linear layer for the final prediction task. The linear layer weights up the evidence from each of these $\mathrm{n}$-grams and make a final decision. Figure 1 shows the basic $\mathrm{CNN}$ operation over an example sentence.

\subsubsection{Training Details}

For the classification task, training is performed for 100 epochs with a batch size of 16 . Adam optimizer is used with a learning rate of 0.001 . Model with the lowest validation loss is saved and

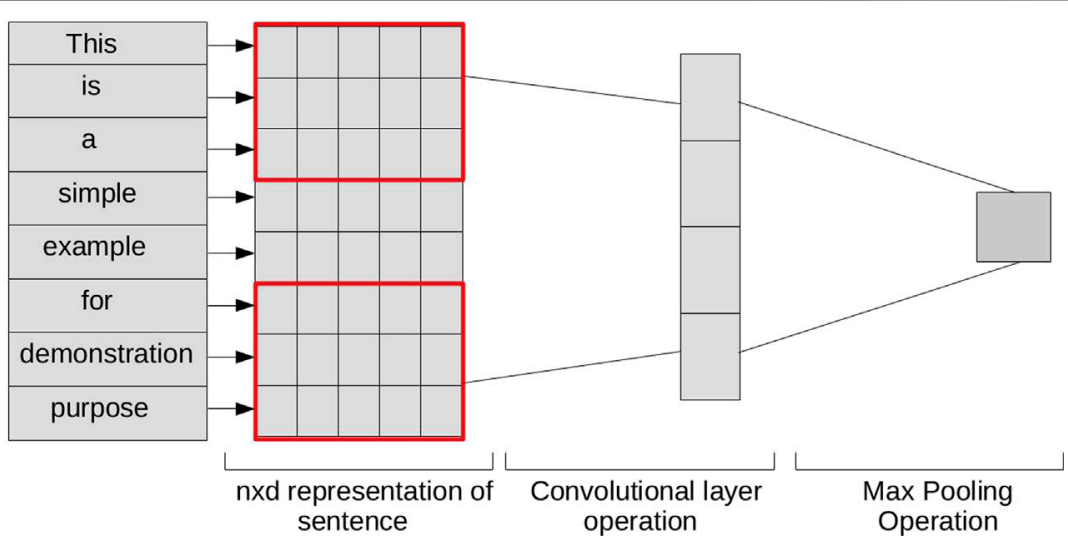

FIGURE 1 | Demonstration of CNN over text for an example sentence. 


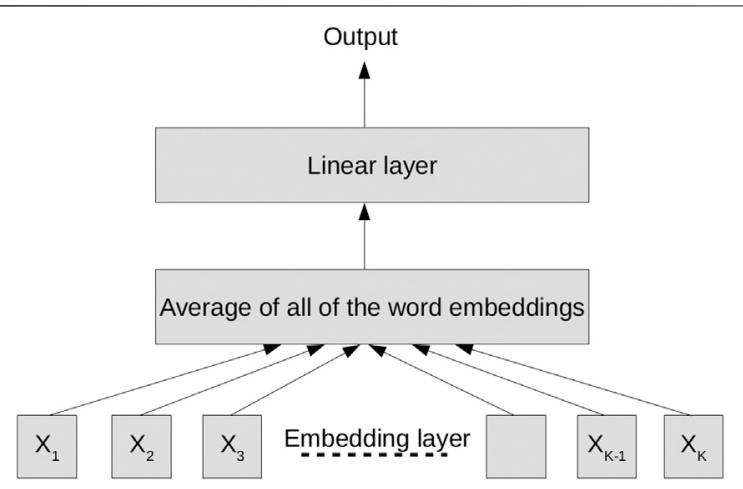

FIGURE 2 | fastText model (Joulin et al., 2017) with appended n-gram features $\left(X_{1}, X_{2}, X_{3}, \ldots, X_{K-1}, X_{K}\right)$ as input.

used for prediction. Since AD classification is a two-class problem, binary cross-entropy with logits loss is used as the loss function. For the MMSE score prediction task, the output layer is a fully connected layer with linear activation function. In the regression task the network is trained for 1,500 epochs with the objective to minimize the mean squared error.

We use bootstrap aggregation of models known as bagging (Breiman, 1996) to predict the final labels/MMSE scores for test samples. Bootstrap aggregation is an ensemble technique to improve the stability and accuracy of machine learning models. It combines the prediction from multiple models. It also reduces variance and helps to avoid overfitting. We fit 21 models and the outputs are combined by a majority voting scheme for final classification. In the regression task, the outputs of these bootstrap models are averaged to arrive at the final MMSE score.

\section{3 fastText}

fastText-based classifiers calculate the n-grams of an input sentence explicitly and append them to the end of the sentence. In this work, we use bigrams and trigrams. We conducted the experiments with 4-grams as well, but the results did not show any improvement over the use of trigrams. This bag of bigrams and trigrams acts as additional features to capture some information about the local word order.

Figure 2 shows the architecture of fastText model. The fastText model has two layers, an embedding layer and a linear layer. The embedding layer calculates the word embedding (100-dimensional) for each word. The average of all these word embeddings is calculated and fed through the linear layer for final prediction as described in Figure 2. fastText models are faster for training and evaluation by many orders of magnitude, compared to the "deep" models. As mentioned in the work (Joulin et al., 2017), fastText can be trained on more than one billion words in less than 10 min using a standard multicore CPU and classify half a million sentences among $312 \mathrm{~K}$ classes in less than a minute.

\subsubsection{Training Details}

All training details are the same as mentioned in Section 4.2.1. The only difference is that dropout is not used in this model. Here
TABLE 2 | Average 5-fold cross-validation results for AD classification and RMSE values.

\begin{tabular}{llcc} 
Dataset & \multicolumn{1}{c}{ Model } & Accuracy & RMSE \\
\hline PAR & CNN, bi+tri+4 gram & 73.91 & 4.55 \\
PAR & CNN, tri+4+5 gram & 77.54 & 4.41 \\
PAR & CNN, bi+tri+4+5 gram & 76.54 & 4.65 \\
PAR & fastText, bigram & 80.54 & 5.43 \\
PAR & fastText, bi + trigram & 82.36 & 5.40 \\
PAR + INV & CNN, bi+tri+4 gram & 80.18 & 4.63 \\
PAR + INV & CNN, tri+4+5 gram & 81.27 & 4.53 \\
PAR + INV & CNN, bi+tri+4+5 gram & 80.36 & 4.38 \\
PAR + INV & fastText, bigram & 86.09 & 4.66 \\
PAR + INV & fastText, bi + trigram & 85.90 & 4.81 \\
\hline
\end{tabular}

also we use 21 bootstrapping models and the outputs are combined as described in Section 4.2.1.

\section{RESULTS}

We have performed 5-fold cross-validation, to estimate the generalization error. One of the folds has 20 validation samples and the remaining four have 22 validation samples. The results of cross-validation on $\mathrm{CNN}$ and fastText models trained on PAR and PAR + INV sets are listed in Table 2 . The best performing model for classification during the crossvalidation was fastText with bigrams on the PAR + INV set, which yields an average cross-validation accuracy of $86.09 \%$. Among the CNN models, tri+4+5 grams give the best accuracy in both PAR (77.54\%) and INV + PAR (81.27\%) sets. As far as accuracy is concerned, both the CNN and fastText models seem to benefit from the inclusion of utterances from the investigator. For the prediction of MMSE score, CNN with bi+tri+4+5 grams (RMSE of 4.38) was the best. The fastText models seem to get a clear advantage in RMSE with the addition of the utterances from the investigator. However such a large difference in RMSE is not observable between the CNN models using PAR and INV + PAR sets. The crossvalidation results confirmed our belief that the n-grams from the transcriptions of the picture description task could be useful in the detection of $\mathrm{AD}$.

Table 3 lists the classification accuracy and RMSE in the prediction of MMSE score on the test set of the ADReSS corpus. The table also lists the precision, recall, and $F_{1}$ score for each class. They are computed as precision $\pi=(T P /(T P+F P))$, recall $\rho=(T P / T P+F N)$, and $F_{1}$ score $=(2 \pi \rho /(\pi+\rho))$, where $T P$, $F P, T N$, and $F N$ are the number of true positives, false positives, true negatives, and false negatives, respectively. The listed results are obtained after bootstrapping with 21 samples. The best classification accuracy is $83.33 \%$ which is achieved using fast Text model with appended bigrams and trigrams. The accuracies are similar in both PAR and PAR + INV sets using the fast Text model. The maximum accuracy obtained with CNN models is $79.16 \%$, which is achieved on the INV + PAR set using bi+tri 4 grams or tri $+4+5$ grams. In the detection task, the CNN models seem to benefit from the addition of utterances from the investigator. Also the accuracies seem to degrade when bigrams, 
TABLE 3 | Results on ADReSS test set. The bold values represent the best results obtained by our models.

\begin{tabular}{|c|c|c|c|c|c|c|c|}
\hline Dataset & Model & Class & Precision & Recall & F1 score & Accuracy (\%) & RMSE \\
\hline \multirow[t]{2}{*}{ PAR } & \multirow[t]{2}{*}{ CNN, bi+tri+4 gram } & Non-AD & 0.74 & 0.71 & 0.72 & \multirow{2}{*}{72.91} & \multirow{2}{*}{4.38} \\
\hline & & $A D$ & 0.72 & 0.75 & 0.73 & & \\
\hline \multirow[t]{2}{*}{ PAR } & \multirow[t]{2}{*}{ CNN, tri+4+5 gram } & Non-AD & 0.76 & 0.67 & 0.71 & \multirow{2}{*}{72.91} & \multirow{2}{*}{4.46} \\
\hline & & $\mathrm{AD}$ & 0.70 & 0.79 & 0.75 & & \\
\hline \multirow[t]{2}{*}{ PAR } & \multirow[t]{2}{*}{$\mathrm{CNN}$, bi+tri+4+5 gram } & Non-AD & 0.71 & 0.71 & 0.71 & \multirow{2}{*}{70.83} & \multirow{2}{*}{4.42} \\
\hline & & $\mathrm{AD}$ & 0.71 & 0.71 & 0.71 & & \\
\hline \multirow[t]{2}{*}{ PAR } & \multirow[t]{2}{*}{ fastText, bigram } & Non-AD & 0.78 & 0.88 & 0.82 & \multirow{2}{*}{81.25} & \multirow{2}{*}{4.51} \\
\hline & & $\mathrm{AD}$ & 0.86 & 0.75 & 0.80 & & \\
\hline \multirow[t]{2}{*}{ PAR } & \multirow[t]{2}{*}{ fastText, bi + trigram } & Non-AD & 0.81 & 0.88 & 0.84 & \multirow{2}{*}{83.33} & \multirow{2}{*}{4.87} \\
\hline & & $A D$ & 0.86 & 0.79 & 0.83 & & \\
\hline \multirow[t]{2}{*}{$P A R+I N V$} & \multirow[t]{2}{*}{ CNN, bi+tri+4 gram } & Non-AD & 0.77 & 0.83 & 0.80 & \multirow{2}{*}{79.16} & \multirow{2}{*}{4.48} \\
\hline & & $A D$ & 0.82 & 0.75 & 0.78 & & \\
\hline \multirow{2}{*}{$P A R+I N V$} & \multirow{2}{*}{ CNN, tri+4+5 gram } & Non-AD & 0.77 & 0.83 & 0.80 & \multirow{2}{*}{79.16} & \multirow{2}{*}{4.47} \\
\hline & & $A D$ & 0.82 & 0.75 & 0.78 & & \\
\hline \multirow[t]{2}{*}{$P A R+I N V$} & \multirow[t]{2}{*}{$\mathrm{CNN}, \mathrm{bi}+\mathrm{tri}+4+5$ gram } & Non-AD & 0.74 & 0.71 & 0.72 & \multirow{2}{*}{72.91} & \multirow{2}{*}{4.44} \\
\hline & & $A D$ & 0.72 & 0.75 & 0.73 & & \\
\hline \multirow[t]{2}{*}{$P A R+I N V$} & \multirow[t]{2}{*}{ fastText, bigram } & Non-AD & 0.78 & 0.88 & 0.82 & \multirow{2}{*}{81.25} & \\
\hline & & $A D$ & 0.86 & 0.75 & 0.80 & & 4.28 \\
\hline$P A R+I N V$ & fastText, bi + trigram & Non-AD & 0.79 & 0.92 & 0.85 & 83.33 & 4.47 \\
\hline & & $A D$ & 0.90 & 0.75 & 0.82 & 00.00 & 4.41 \\
\hline
\end{tabular}

trigrams, 4-grams, and 5-grams are considered together. This behavior is consistent across the PAR and PAR + INV sets. The best RMSE in the prediction of MMSE score is 4.28 which is obtained on the PAR + INV set using fastText model employing only bigrams. In the regression task using fastText, the use of bigrams achieves slightly better RMSE compared to the use of both bigrams and trigrams. Also the fastText models seem to benefit from the use of utterances from the investigator. In contrast, CNN models do not seem to get any specific advantage with the inclusion of investigator's utterances. The performance of the $\mathrm{CNN}$ models remains almost the same across the use of bi+tri +4 , tri $+4+5$, and bi+tri $+4+5$ grams.

\section{DISCUSSION AND CONCLUSION}

In this work, we explore two models, $\mathrm{CNN}$ with a single convolution layer and fastText, to address the problem of $\mathrm{AD}$ classification and prediction of MMSE score from the transcriptions of the picture description task. The choice of these models was based on our initial belief that modeling the transcriptions of the narrative speech in the picture description task using n-grams could give some indication on the status of $\mathrm{AD}$. The chosen models are also shallow. The number of parameters is much less than the usual deep learning architectures and hence they can be trained and evaluated quite fast. Yet, the performance of these models is competitive with the baseline results reported with complex models (refer to Table 1). The results suggest that the n-gram-based features are worth pursuing, for the task of AD detection.

Among the considered models, fastText model with bigrams and trigrams appended to the input achieves the best classification accuracy $(83.33 \%)$. In the regression task, the best results (RMSE of 4.28) are achieved using fastText model with only the bigrams appended to the input. The fastText models have a clear edge over CNN in the classification task. Empirical evidence suggests that fastText models benefit from the inclusion of utterances from the investigator in the regression task, though they do not make much difference in the classification task. The CNN models on the other hand perform better on the PAR + INV sets in the classification task. In the regression task, their performance is similar across the PAR and PAR + INV sets. Bigrams have an edge over bi + tri grams in fastText, when used for prediction of MMSE score. However, the performance of the CNN models remains almost the same across the use of bi+tri+4, tri $+4+5$, and bi+tri $+4+5$ grams, in the regression task.

\section{DATA AVAILABILITY STATEMENT}

The data analyzed in this study are subject to the following licenses/restrictions: In order to gain access to the ADReSS data, you will need to become a member of DementiaBank (free of charge) by contacting Brian MacWhinney on macw@cmu.edu. You should include your contact information and affiliation, as well as a general statement on how you plan to use the data, with specific mention to the ADReSS challenge. If you are a student, please ask your supervisor to join as a member as well. This membership will give you full access to the DementiaBank database, where the ADReSS dataset will be available and clearly identified. For further information, visit DementiaBank. Requests to access these datasets should be directed to Brian MacWhinney, macw@cmu.edu.

\section{AUTHOR CONTRIBUTIONS}

AM, AS, and AR contributed to the conception and design of the study. AM and AS wrote the first draft of the manuscript. AR reviewed the first draft and suggested improvements. AM and AS wrote sections of the manuscript. All authors contributed to manuscript revision and read and approved the submitted version. 


\section{REFERENCES}

Breiman, L. (1996). Bagging predictors. Mach. Learn. 24, 123-140. doi:10.1007/ BF00058655

Campbell, E. L., Docío-Fernández, L., Raboso, J. J., and García-Mateo, C. (2020). Alzheimer's dementia detection from audio and text modalities. arXiv preprint arXiv:2008.04617

Chen, J., Wang, Y., and Wang, D. (2014). A feature study for classification-based speech separation at low signal-to-noise ratios. IEEE/ACM Trans. Audio Speech Lang. Process. 22, 1993-2002. doi:10.1109/TASLP.2014.2359159

Collober, R., Weston, J., Bottou, L., Karlen, M., Kavukcuoglu, K., and Kuksa, P. (2011). Natural language processing (almost) from scratch. J. Machine Learn. Res. 12, 2493-2537. doi:10.5555/1953048.2078186

Cong, J., and Liu, H. (2014). Approaching human language with complex networks. Phys. Life Rev. 11, 598-618. doi:10.1016/j.plrev.2014.04.004

Corra, E. A., Lopes, A. A., and Amancio, D. R. (2018). Word sense disambiguation. Inf. Sci. 442, 103-113. doi:10.1016/j.ins.2018.02.047

Dai, Z., Yang, Z., Yang, Y., Carbonell, J., Le, Q., and Salakhutdinov, R. (2019). "Transformer-XL: attentive language models beyond a fixed-length context," in Proceedings of the 57th annual meeting of the association for computational linguistics, Florence, Italy, July 2019, 2978-2988. doi:10.18653/v1/P19-1285

De Arruda, H., Costa, L., and Amancio, D. (2016). Using complex networks for text classification: discriminating informative and imaginative documents. EPL 113, 28007. doi:10.1209/0295-5075/113/28007

Devlin, J., Chang, M.-W., Lee, K., and Toutanova, K. (2019). "BERT: pre-training of deep bidirectional transformers for language understanding," in Proceedings of the 2019 conference of the north american chapter of the association for computational linguistics: human language technologies, Minneapolis, MN, June 2-7, 2019, Vol. 1, 4171-4186. doi:10.18653/v1/N19-1423

Edwards, E., Dognin, C., Bollepalli, B., and Singh, M. (2020). "Multiscale system for Alzheimer's dementia recognition through spontaneous speech," in Proceedings of interspeech 2020, Shanghai, China, October 2020, 2197-2201. doi:10.21437/Interspeech.2020-2781

Eyben, F., Scherer, K. R., Schuller, B. W., Sundberg, J., André, E., Busso, C., et al. (2016). The Geneva minimalistic acoustic parameter set (GeMAPS) for voice research and affective computing. IEEE Trans. Affective Comput. 7, 190-202. doi:10.1109/taffc.2015.2457417

Eyben, F., Weninger, F., Gross, F., and Schuller, B. (2013). "Recent developments in openSMILE, the Munich open-source multimedia feature extractor," in Proceedings the 2013 ACM multimedia conference, Barcelona, Spain, October, 2013, 835-838. doi:10.1145/2502081.2502224

Gemmeke, J., Ellis, D., Freedman, D., Jansen, A., Lawrence, W., Moore, R., et al. (2017). "Audio set: an ontology and human-labeled dataset for audio events," in 2017 IEEE international conference on acoustics, speech and signal processing (ICASSP), New Orleans, LA, March 5-9, 2017, 776-780. doi:10.1109/ICASSP. 2017.7952261

Hershey, S., Chaudhuri, S., Ellis, D., Gemmeke, J., Jansen, A., Moore, R. C., et al. (2017). "CNN architectures for large-scale audio classification," in 2017 IEEE international conference on acoustics, speech and signal processing (ICASSP), New Orleans, LA, March 5-9, 2017, 131-(135.)

Joulin, A., Grave, E., Bojanowski, P., and Mikolov, T. (2017). "Bag of tricks for efficient text classification," in Proceedings of the 15th conference of the european chapter of the association for computational linguistics, Valencia, Spain, April 3-7, 2017, Vol. 2, 427-(431.)

Kim, Y. (2014). "Convolutional neural networks for sentence classification," in Proceedings of the 2014 conference on empirical methods in natural language processing (EMNLP), Doha, Qatar, October 25-29, 2014, 1746-1751. doi:10. 3115/v1/D14-1181

Koo, J., Lee, J. H., Pyo, J., Jo, Y., and Lee, K. (2020). “Exploiting multi-modal features from pre-trained networks for Alzheimer's dementia recognition," in Proceedings of interspeech 2020, Shanghai, China, October 2020, 2217-2221. doi:10.21437/Interspeech.2020-3153

Liu, Y., Ott, M., Goyal, N., Du, J., Joshi, M., Chen, D., et al. (2019). RoBERTa: a robustly optimized BERT pretraining approach. ArXiv abs/1907.11692

Luz, S., Haider, F., de la Fuente, S., Fromm, D., and MacWhinney, B. (2020). "Alzheimer's dementia recognition through spontaneous speech: the ADReSS challenge," in Proceedings of interspeech 2020, Shanghai, China, October 2020, 2172-2176. doi:10.21437/Interspeech.2020-2571

Macwhinney, B. (2009). "The CHILDES project part 1," in The CHAT transcription format. doi:10.1184/R1/6618440.v1

Meghanani, A., Anoop, C. S., and Ramakrishnan, A. G. (2021). “An exploration of log-mel spectrogram and MFCC features for Alzheimer's dementia recognition from spontaneous speech," in The 8th IEEE spoken language technology workshop (SLT), Shenzhen, China, January 19-22, 2021

Mueller, K. D., Hermann, B., Mecollarib, J., and Turkstra, L. S. (2018a). Connected speech and language in mild cognitive impairment and Alzheimer's disease: a review of picture description tasks. J. Clin. Exp. Neuropsychol. 40, 917-939. doi:10.1080/13803395.2018.1446513

Mueller, K. D., Koscik, R. L., Hermann, B., Johnson, S. C., and Turkstra, L. S. (2018b). Declines in connected language are associated with very early mild cognitive impairment: results from the Wisconsin registry for Alzheimer's prevention. Front. Aging Neurosci. 9, 437. doi:10.3389/fnagi.2017.00437

Nicholas, M., Obler, L. K., Albert, M., and Helm-Estabrooks, N. (1985). Empty speech in Alzheimer's disease and fluent aphasia. J. Speech Hear. Res. 28, 405-410. doi:10.1044/jshr.2803.405

Pappagari, R., Cho, J., Moro-Velázquez, L., and Dehak, N. (2020). "Using state of the art speaker recognition and natural language processing technologies to detect Alzheimer's disease and assess its severity," in Proceedings of interspeech 2020, Shanghai, China, October 2020, 2177-2181. doi:10.21437/Interspeech.2020-2587 Pennington, J., Socher, R., and Manning, C. (2014). Glove: global vectors for word representation. Proceedings of the 2014 conference on empirical methods in natural language processing (EMNLP), Doha, Qatar, October 25-29, 2014, 1532-1543. doi:10.3115/v1/d14-1162

Pompili, A., Rolland, T., and Abad, A. (2020). "The INESC-ID multi-modal system for the ADReSS 2020 challenge," in Proceedings of interspeech 2020, Shanghai, China, October 2020, 2202-2206. doi:10.21437/Interspeech.2020-2833

Radford, A., Narasimhan, K., Salimans, T., and Sutskever, I. (2018). Improving language understanding by generative pre-training. Available at: https://www. cs.ubc.ca/amuham01/LING530/papers/radford2018improving.pdf. doi:10. 1017/9781108552202

Rohanian, M., Hough, J., and Purver, M. (2020). "Multi-Modal fusion with gating using audio, lexical and disfluency features for Alzheimer's dementia recognition from spontaneous speech," in Proceedings of interspeech 2020, Shanghai, China, October 2020, 2187-2191. doi:10.21437/Interspeech.2020-2721

Sanh, V., Debut, L., Chaumond, J., and Wolf, T. (2019). DistilBERT, a distilled version of BERT: smaller, faster, cheaper and lighter. ArXiv abs/1910.01108

Santos, L., Corrêa Júnior, E. A., Oliveira, O., Jr., Amancio, D., Mansur, L., and Aluísio, S. (2017). "Enriching complex networks with word embeddings for detecting mild cognitive impairment from speech transcripts," in Proceedings the 55th annual meet: the association for computational linguistics, Vancouver, BC, July 30-August 4, 2017, Vol. 1, 1284-1296. doi:10.18653/v1/P17-1118

Sarawgi, U., Zulfikar, W., Khincha, R., and Maes, P. (2020a). Uncertainty-aware multi-modal ensembling for severity prediction of Alzheimer's dementia. ArXiv abs/2010.01440. doi:10.21437/interspeech.2020-3137

Sarawgi, U., Zulfikar, W., Soliman, N., and Maes, P. (2020b). Multimodal inductive transfer learning for detection of Alzheimer's dementia and its severity. arXiv preprint arXiv:2009.00700. doi:10.21437/interspeech.2020-3137

Savundranayagam, M., Hummert, M. L., and Montgomery, R. (2005). Investigating the effects of communication problems on caregiver burden. J. Gerontol. B Psychol. Sci. Soc. Sci. 60 (1), S48-S55. doi:10.1093/geronb/60.1.s48

Searle, T., Ibrahim, Z., and Dobson, R. (2020). "Comparing natural language processing techniques for Alzheimer's dementia prediction in spontaneous speech," in Proceedings of interspeech 2020, Shanghai, China, October 2020, 2192-2196. doi:10.21437/Interspeech.2020-2729

Shen, Y., He, X., Gao, J., Deng, L., and Mesnil, G. (2014). "Learning semantic representations using convolutional neural networks for web search," in WWW 2014, Seoul, South Korea, April 7-11, 2014, 373-374. doi:10.1145/2567948.2577348

Syed, M. S. S., Syed, Z. S., Lech, M., and Pirogova, E. (2020). “Automated screening for Alzheimer's dementia through spontaneous speech," Proceedings of interspeech 2020, Shanghai, China, October 2020, 2222-2226. doi:10.21437/ Interspeech.2020-3158

Szatlóczki, G., Hoffmann, I., Vincze, V., Kálmán, J., and Pákáski, M. (2015). Speaking in Alzheimer's disease, is that an early sign? Importance of changes in 
language abilities in Alzheimer's disease. Front. Aging Neurosci. 7, 110. doi:10. 3389/fnagi.2015.00195

tau Yih, W., He, X., and Meek, C. (2014). "Semantic parsing for single-relation question answering," in Proceedings of the 52nd annual meeting of the association for computational linguistics, Baltimore, MA, June 2014, Vol. 2, 643-648. doi:10.3115/v1/P14-2105

Tomás, D. R. M., and Radev, D. (2012). Graph-based natural language processing and information retrieval. Machine Translation 26, 277-280. doi:10.1007/ s10590-011-9122-9

Vaswani, A., Shazeer, N., Parmar, N., Uszkoreit, J., Jones, L., Gomez, A., et al. (2017). "Attention is all you need," Proceedings of the 31st international conference on neural information processing systems, Long Beach, CA, December 2017, 5999-(6009.)

Yuan, J., Bian, Y., Cai, X., Huang, J., Ye, Z., and Church, K. (2020). “Disfluencies and fine-tuning pre-trained language models for detection of Alzheimer's disease," in Proceedings of interspeech 2020, Shanghai, China, October 2020, 2162-2166. doi:10.21437/Interspeech.2020-2516

Conflict of Interest: The authors declare that the research was conducted in the absence of any commercial or financial relationships that could be construed as a potential conflict of interest.

Copyright (C) 2021 Meghanani, Anoop and Ramakrishnan. This is an open-access article distributed under the terms of the Creative Commons Attribution License (CC $B Y)$. The use, distribution or reproduction in other forums is permitted, provided the original author(s) and the copyright owner(s) are credited and that the original publication in this journal is cited, in accordance with accepted academic practice. No use, distribution or reproduction is permitted which does not comply with these terms. 